\title{
EL CRETÁCICO SUPERIOR EN EL PIRINEO. PALEONTOLOGÍA
}

\author{
José María PONS ${ }^{1}$ y Esmeralda CAUS ${ }^{1}$ \\ ' Departament de Geología. Universitat Autònoma de Barcelona, 08193 Bellaterra.
}

Pons, J. M. y Caus, E. 1996. El Cretácico Superior en el Pirineo. Paleontología. [The Upper Cretaceous from Pyrenees. Paleontology]. Revista Española de Paleontología, N Extraordinario, 182-189. ISSN 0213-6937.

\begin{abstract}
The contributions to the knowledge of the fossil record, biostratigraphy, palaeoecology, palaeobiogeography and geology of the marine Upper Cretaceous of the Pyrenees, contained in 72 palaeontological papers produced at the Universitat Autonoma de Barcelona, are commented.
\end{abstract}

Keywords: Upper Cretaceous, Pyrenees, Taxonomy, Biostratigraphy, Palaeoecology, Palaeobiogeography, Geology.

\section{RESUMEN}

Se comentan las aportaciones al conocimiento del registro fósil, bioestratigrafía, paleoecología, paleobiogeografía y geología del Cretácico superior marino pirenaico, contenidas en 72 trabajos de paleontología realizados en la Universidad Autònoma de Barcelona.

Palabras clave: Cretácico superior, Pirineos, Taxonomía, Bioestratigrafía, Paleoecología, Paleobiogeografía, Geología.

\section{INTRODUCCIÓN}

El grupo de Paleontología de la Universitat Autònoma de Barcelona viene ocupándose desde 1971 del estudio de los fósiles del Cretácico superior de la zona surpirenaica, concretamente de su parte cenral y extendiéndose seguidamente a su parte oriental (Fig. 1), siguiendo la línea iniciada con la tesis doctoral sobre los rudistas del primero de los firmantes, que fue dirigida por el profesor J. Rosell.

Principalmente después de su organización como Departamento de Paleontología en 1975 y coincidiendo con la irrupción de las primeras promociones de geólogos formados en esta Universidad, se comenzó un conjunto de trabajos de licenciatura, la mayoría estudios descriptivos y taxonómicos destinados a inventariar distintos grupos fósiles y datar los sedimentos que los contienen, pero que no obstante, aportaron datos de valor regional sobre bioestratigrafía, paleoecología y/o paleobiogeografía. Algunos de estos estudios fueron continuados, con objetivos semejantes, como tesis doctorales al mismo tiempo que se iniciaron otras con objetivos más puramente paleoecológicos o bioestratigráficos.

Paralelamente, en diversos trabajos se ha profundizado en algunos aspectos taxonómicos, bioestratigráficos, paleoecológicos y/o paleobiogeográficos de esta fauna, se han presentado síntesis bioestratigráficas parciales, se ha contribuido a la resolución de problemas geológicos regionales y se han realizado estudios comparativos con otras áreas. La colaboración con otros grupos de investigadores y la financiación de proyectos de investigación, aunque no tuvieran como objetivo principal la zona pirenaica, han contribuido de modo decisivo a llevar a buen término muchos de estos trabajos.

Así pues, el objetivo de esta revisión es dar a conocer el estado actual de las investigaciones sobre la paleontología del Cretácico superior pirenaico con un resumen de las apor- taciones a la taxonomía, bioestratigrafía, paleoecología, paleobiogeografía y conocimiento de la geología regional contenidas en todos estos trabajos.

\section{MICROPALEONTOLOGÍA}

Los primeros trabajos realizados en el Departamento de Paleontología de la Universidad Autònoma de Barcelona, en el campo de la Micropaleontología del Cretácico del Pirineo catalán, estuvieron encaminados a conocer áreas particulares ya fuera por su interés paleontológico o bien por su importancia geológica. Fruto de estas primeras investigaciones fueron varios trabajos de licenciatura (Cornella, 1977; Mañas, 1977; Conesa, 1979; Gómez-Garrido, 1981 y Rodés, 1983) y otros (Mañas, Caus y Pons, 1979) donde se aportaron datos sobre los foraminíferos, tanto bentónicos como planctónicos.

Los resultados obtenidos en estos trabajos se completaron con otros destinados principalmente a conocer la edad de los sedimentos y el medio en que se habían depositado. Así, se realizó un trabajo de síntesis sobre los ciclos sedimentarios del Cretácico superior en la cuenca surpirenaica, entre los ríos Segre y Noguera-Ribagorçana (Caus, Cornella y GómezGarrido, 1982) y se estudió la significación paleoambiental y repartición bioestratigráfica de los macroforaminíferos de la Sierra del Montsec (Caus y Cornella, 1983), con objeto de hacer un análisis de la historia deposicional del Cretácico superior de la región.

Los estudios no se centraron únicamente en el área surpirenaica central sino que se ampliaron al área situada al E del río Segre (Sierras de Turb y Port de Compte) donde se realizó una cartografía de detalle, se estudiaron los foraminíferos y se establecieron las bases biocronoestratigráficas para la interpretación tectónica (Caus, Rodés y Solé, 1988). En el 


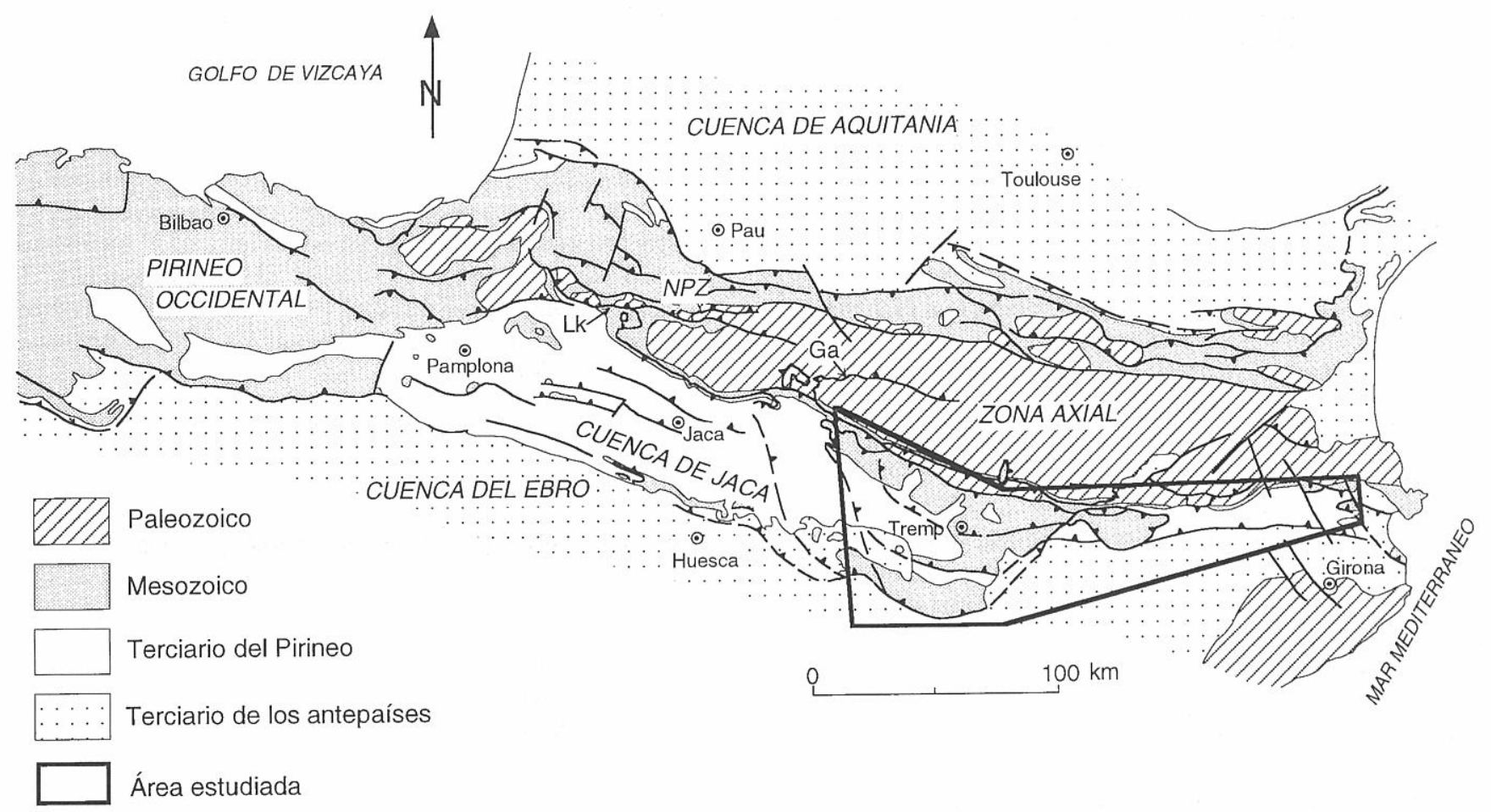

Figura 1. Localización del área estudiada sobre un esquema tectónico de la cordillera pirenaica tomado de Teixell (1992).

área del Alt Empordà, la realización de un rabajo de licenciatura sobre los rudistas (Vicens, 1984) permitió conocer la presencia de una importante forma de foraminífero aporcelanado, Larrazetia larrazeti (Munier-Chalmas) presentando un botón umbilical formado por superposición de las prolongaciones alares de cada cámara (Caus y Vicens, 1985). La datación de los niveles conteniendo dicho foraminífero como del Santoniense superior ha sido posible por la presencia en los mismos estratos de Praesiderolites santoniensis Wannier y Orbitoides hottingeri Van Hinte. Sedimentos situados superiormente a los indicados y conteniendo Praelacazina fragilis (Hofker) ("Rzehakinella" n. gen. en Caus y Vicens, 1985) y Adrahentina iberica Bilotte han sido datados del Campaniense medio por la fauna de Orbitoides (O. tissoti (Schlumberger)).

Los trabajos, principalmente bioestratigráficos, basados en los foraminíferos proporcionaron importante información y material sobre los mismos, permitiendo emprender un conjunto de estudios ecaminados a conocer la significación funcional de la morfología y estructura de los foraminíferos, así como la posición sistemática de los mismos. Caus, Cornella y Pons (1978), en un estudio sobre los macroforaminíferos de la zona de Tragó de Noguera (Sierras Marginales), estudian la estrucutra de "Dictyopsella" chalmasi Schlumberger constatando que la citada especie no corresponde al género Dictyopsella MunierChalmas y debe ser adscrita al género Hemicyclammina Maync ya que presenta los hemiseptos típicos del citado género.

Entre los macroforaminíferos aglutinados de las facies poco profundas del Santoniense de la Sierra de Montsec se describió una nueva especie del género Montsechiana Aubert et al., M. montsechiensis (Caus y Cornella, 1981a), que se diferencia de la especie-tipo del género, M. martiguae Aubert et al., por la presencia de un endoesqueleto formado por pila- res radiales continuos pero alternantes de una cámara a la siguiente en sucesivos planos de estolones. Se destaca también la presencia en el género Montsechiana de un exoesqueleto formado por vigas y "pseudoporos", por lo que su origen debería encontrarse entre los pequeños foraminíferos aglutinados simples.

El descubrimiento de un foraminífero cónico con estructura "orbitoliniforme" (Caus y Cornella, 1981b), en facies campanienses poco profundas y escasa energía del agua, permite señalar el rol ecológico marginal jugado por los sucesores de los Orbitolínidos s. str. El nuevo género, Calvecico$n u s$, se caracteriza por la presencia de un exoesqueleto formado por vigas y viguecillas, pero careciendo de epidermis, análogo al del género terciario Fallotella, y por un endoesqueleto constituido por tabiques radiales ondulados, con estolones entrecruzados típicamente orbitolinoformes.

Ilerdorbis (Hottinger y Caus, 1982) es también un nuevo género descrito de las mismas facies, pero su morfología es de planispiral evoluta a cíclica, con un sistema de estolones oblicuo entrecruzado. Un tipo de endoesqueleto tal, en foraminíferos aglutinados, era conocido solo en formas orbitoliniformes cónicas con una disposición de las camaras uniseriada. La presencia de este tipo de endoesqueleto en formas aglutinadas discoidales demuestra que los modelos endoesqueléticos son independientes de la disposición de las cámaras y aparecen, en diferentes épocas, geológicas, en diferentes grupos taxonómicos. En consecuencia, se propuso dos nuevas familias de foraminíferos discoidales aglutinados: Ilerdorbinae, caracterizada por conchas finamente aglutinadas con un modo de crecimiento peneropliforme-discoidal, exoesqueleto simple y un sistema de estolones entrecruzado, comprendiendo los géneros Ilerdorbis y Dohaia, y Orbitop- 
sellinae con el género Orbitopsella como tipo y que se diferenciaría del anterior por un sistema radial de disposición de los ejes de los estolones.

La distribución ambiental de los macroforaminíferos del Cretácico superior surpirenaico queda reflejada en dos trabajos sucesivos (Caus y Serra-Kiel, 1984 y Caus, 1988) donde, además de su distribución en las distintas zonas de la plataforma, se hace referencia al tipo de plataforma, carbonatada o terrígena, y se comparan las estructuras que presentan los distintos grupos de foraminíferos en relación con el sustrato y la energía del agua, principalmente.

La necesidad de realizar una bioestratigrafía detallada de las secuencias deposicionales y la correlación entre los sedimentos de plataforma nerítica y talud y/o cuenca del Cretácico superior del Pirineo hace necesario poner más énfasis en los estudios sobre foraminíferos planctónicos. Resultado de ello es la realización de una tesis doctoral (Gómez-Garrido, 1988) y numerosos estudios que representan no sólo aportaciones al conocimiento de la bioestratigrafía de las facies de foraminíferos planctónicos del Cretácico (GómezGarrido, 1989) sino también estudios de correlación que implican unas escalas comparadas de foraminíferos planctónicos y bentónicos (Caus y Gómez-Garrido, 1989a y 1989b, Caus et al. 1993). Ello permite realizar una correlación entre los sedimentos de los mantos de Boixols, Montsec y Sierras Marginales, representando respectivamente las facies de cuenca y talud, plataforma abierta/plataforma protegida y plataforma poco profunda/plataforma restringida (Caus y Gómez-Garrido, 1989b), las secuencias deposicionales y las unidades litoestratigráficas.

La discusión existente acerca de la edad de los sedimentos marinos superiores (Maastrichtiense sensu lato) en el Pirineo meridional, llevó a estudiar en detalle la repartición de las especies del género Lepidorbitoides (Caus, Gómez-Garrido y Rodés, 1988), llegándose a la conclusión que L. bisambergensis era típico del Maastrichtiense basal (zona de Globotruncana falsostuarti) mientras que entre los representantes pirenaicos que se atribuían a la especie $L$. socialis había varias poblaciones diferentes, la población más inferior es correlacionable con la parte superior de la zona de G. falsostuarti mientras que las poblaciones superiores, presentes sólo en el Pirineo septentrional, son correlacionables, respectivamente, con las zonas de Gansserina gansseri y Abathomphalus mayaroensis, de edad Maastrichtiense medio y superior. La población de Maastricht corresponde a una especie distinta, $L$. minor, no presente en el Pirineo.

La comparación de la fauna pirenaica con la de otras áreas del Mediterráneo pone de manifiesto que el golfo pirenaico, que se extendía desde la plataforma cantábrica hasta cerca de Marsella, llegando por el sur hasta el borde norte de las cordilleras Béticas, contiene una fauna de macroforaminíferos endémica; el análisis de la citada fauna permite poner de manifiesto que los macroforaminíferos neófitos después del Turoniense, con una estrategia de vida $\mathrm{K}$ extrema y viviendo en la parte superior de la zona eufótica (Meandropsínidos, Fabuláridos y Alveolínidos principalmente) desarrollaron formas endémicas más fácilmente que los macroforaminíferos de tipo orbitoidal que vivían en aguas más profundas (Caus y Hottinger, 1986).

El inventario de los Miliólidos trematoporados de la provincia pirenaica (Hottinger, Drobne y Caus, 1989) pone de manifiesto la existencia de nuevas especies: Pseudolacazina loeblichii, P. cantabrica, Lacazina pyrenaica y L. elongata ovula. Igualmente se estudia una forma globosa, identificada hasta entonces como un representante de los Fabuláridos, llegándose a la conclusión de que se trata de un nuevo alveolínido santoniense, Hellenalveolina tappanae.

En los sedimentos neríticos profundos campanienses y maastrichtienses existe una forma ubiquista y fácilmente identificable, Praestorrsella roestae. El estudio detallado de su estructura (Hottinger y Caus, 1993) ha permitido transferir la citada especie de la familia Rotaliidae a Gabratellidae.

\section{MACROPALEONTOLOGÍA}

Son diversos los grupos de macrofauna estudiados y no todos fueron tratados en igual profundidad ni bajo el mismo aspecto.

\section{Corales}

Los escleractínidos del Santoniense de les Collades de Basturs fueron el objeto de estudio de una tesis de licenciatura. Vidal (1980) describió 33 especies de las que 27 se citaban por primera vez en la localidad y 16 en Cataluña. Para todas las especies se da la descripción de las estructuras externas e internas, tablas de medidas y gráficos de variabilidad, se incluyen dibujos a cámara clara de las estructuras internas y fotografías en superficie y en sección. Después de un estudio de las morfologías más frecuentes y su localización en los distintos niveles del yacimiento se apuntan una serie de conclusiones de tipo paleoecológico. El estudio de los corales cretácicos ha sido reemprendido recientemente en el marco de la tesis doctoral.

\section{Inocerámidos}

Los bivalvos inocerámidos de los alrededores de Sant Corneli fueron el tema de otra tesis de licenciatura. López (1986a) describió 35 taxones de inocerámidos, haciendo especial énfasis en la variación ontogénica, presentada en gráficas. Sus conclusiones son principalmente bioestratigráficas, presentando 8 asociaciones de inocerámidos referidas a las zonas de ammonites reconocidas en la misma área, desde el Coniaciense al Maastrichtiense inferior. López (1986b) mostró la distribución de los inocerámidos en la zona surpirenaica central. La tesis doctoral no la realizó en Pirineos sino en la cuenca navarro-cantábrica, pero presentó (López, Martínez y Lamolda, 1992) una comparación de la distribución estratigráfica de los inocerámidos del Coniaciense y Santoniense en las dos cuencas. López (en prensa) describe los inocerámidos de la sierra del Montsec.

\section{Rudistas}

El estudio de los bivalvos rudistas se inició con la tesis doctoral del primero de los firmantes; Pons (1977) describió 68 especies de rudistas del Coniaciense al Maastrichtiense de las Sierras Marginales, la Sierra del Montsec y el $\mathrm{N}$ de la Conca de Tremp. Como conclusión al estudio estratigráfico y paleontológico, correlacionó los yacimientos de 
las tres áreas y estableció 5 cenozonas del Coniaciense-Santoniense inferior al Maastrichtiense inferior. Pons (1982) presentó la distribución de los rudistas del Cretático superior surpirenaico por pisos, del Cenomaniense al Maastrichtiense, y por áreas geográficas, Sierras Marginales, Montsec, N de la Conca de Tremp, Segre-Freser y Girona. Philip, Bilotte y Pons, (1983) presentaron la distribución de los rudistas basada en datos de ambas vertientes del Pirineo, Aquitania y Provenza.

Una tesis de licenciatura (Sánchez, 1981), consistió en la elaboración del catálogo de especies de Hippuritidae y Radiolitidae, recogiendo todas las citas hasta la fecha. Otra, emprendía el estudio de los yacimientos con rudistas del Alt Empordà; Vicens (1984) describió 22 especies y distinguió 2 asociaciones, una campaniense inferior y otra campaniense superior.

Un estudio de la estructura de la concha de Biradiolites chaperi Bayle (Pons y Vicens, 1986), puso en sinonimia con esta especie varias formas consideradas endémicas de la bioprovincia mediterránea oriental; este hecho, junto al hallazgo en Pirineos de otras especies también "orientales" permitió señalar las deficiencias de los datos en que se basaban las provincias paleobiogeográficas del Tethys (Gili, Pons y Vicens, 1987). Pons y Vicens (1991) señalaron las diferencias entre la fauna campaniense y maastrichtiense de Pirineos y la del sur de la provincia de Valencia, de afinidades tethysianas. Pons y Sirna (1992) analizaron las semejanzas y diferencias entre las faunas de rudistas de España (Pirineos y Valencia) e Italia centro meridional en función de la distinta composición y distribución de facies, causadas por la diversidad de tipos de plataformas y la evolución geodinámica de esta parte del Tethys.

Gili, Pons y Vicens (1986) plantearon la problemática del uso de los rudistas en bioestratigrafía, basándose en la experiencia adquirida en su aplicación a la zona pirenaica. Pascual, Pons y Vicens (1989) presentaron la distribución estratigráfica de los rudistas desde el Turoniense al Maastrichtiense sobre una serie estratigráfica estudiada en detalle en el Montsec, distinguiendo 9 asociaciones sucesivas, una turoniense, una coniaciense, dos santonienses, tres campanienses y dos maastrichtienses, que han servido de escala para aplicar a otras zonas pirenaicas de mayor complejidad tectónica.

En su tesis de doctorado, Vicens (1992a) estudió los yacimientos con rudistas del Pirineo oriental, describió 21 especies de hippurítidos y 30 de radiolítidos procedentes de las 2 unidades del manto inferior del Pedraforca, de la unidad Cadí y de la unidad Port del Compte; en las conclusiones bioestratigráficas reconoció, desde el Santoniense superior al Maastrichtiense inferior, las asociaciones distinguidas por Pascual, Pons y Vicens (1989), distinguiendo además una asociación Ca-Ma que comprendería parte de la última asociación campaniense y de la primera maastrichtiense. Vicens (1992b) presentó las conclusiones relativas a los hippurítidos de su tesis doctoral.

Las interacciones sedimentológicas y biológicas en las formaciones de rudistas del Santoniense de les Collades de Basturs fue el tema de otra tesis de doctorado (Gili, 1983); además, en diversos trabajos (Gili, 1982, 1992a, 1993; Gili y Pons, 1983 y 1988) y guías de excursiones (Gili, 1985, 1992b) se ha ido afinando cada vez más el análisis sedimen- tológico y la distribución de los rudistas en estas formaciones que en Scott et al. (1990) fueron tomadas como un ejemplo de arrecife de corales y rudistas en el Cretácico superior.

\section{Ammonites}

La primera tesis de licenciatura sobre ammonites versó sobre los del Aptiense superior de la formación Margas de Llucà, entonces considerados del Aptiense-Albiense. Martínez (1977) describió 19 especies de ammonites, determinó la fauna acompañante en los yacimientos considerados y discutió los problemas tafonómicos y bioestratigráficos planteados por la fauna; Martínez (1979) discutió algunos aspectos de las especies más representativas y añadió 5 especies más al inventario. En sus tesis doctoral, Martínez (1982a) describió 131 especies de ammonites, del Aptiense inferior al Maastrichtiense inferior, de la zona comprendida entre el río Segre y el Noguera-Ribagorçana. Como conclusión bioestratigráfica presentó la distribución de estas especies en 18 biozonas; esta biozonación ha servido de referencia para la datación de otra macrofauna cretácica pirenaica. Martínez (1982b) resumió y comentó esta zonación. Martínez y Vicens (1987-88) describieron 15 especies de ammonites del Campaniense y Maastrichtiense de la Unidad Pedraforca.

\section{Braquiópodos}

Aparte de un primer trabajo de Gallemí y Calzada (1979) en que se describe la nueva especie "Rhynchonella" coquandi, el estudio de los braquiópodos también empezó con una tesis de licenciatura. Muñoz (1985) además de la descripción de los caracteres externos e internos de 13 especies de los alrededores de Sant Corneli realizó, gracias al considerable número de ejemplares estudiados, un estudio estadístico de la fauna de rinconéllidos y terebratúlidos por unidades litoestratigráficas; presentó la distribución estratigráfica de las especies referidas a las zonaciones de ammonites y resaltó la semejanza de su fauna con la de Aquitania y las diferencias con la del sur de Inglaterra.

Muñoz (1990) inició el estudio de los microbraquiópodos con la descripción de 8 especies del Bergadá y planteó el problema de las formas monoseptales dentro de los Thecideida. En su tesis doctoral, Muñoz (1993) estudió los braquiópodos del Cretácico superior pirenaico abarcando las áreas de las Sierras Marginales, de la Sierra del Montsec, de Tremp, de Berga y de Girona. Describe 40 especies, proponiendo 8 nuevas, 4 nuevos géneros y 2 nuevas subfamilias. La distribución estratigráfica se presenta por áreas y referida a las dataciones obtenidas con otros grupos faunísticos y se adjuntan unos cuadros de distribución/abundancia para cada orden de braquiópodos. Muñoz (1994) describe Mesoseptina minima, nuevo género y especie de Thecidellininae de la Sierra del Montsec, permitiéndole aclarar las relaciones filéticas de las formas de tecideas con el aparato monoseptal cerrado y simple.

\section{Equínidos}

La tesis de licenciatura que inició los estudios sobre los equínidos cretácicos (Gallemí, 1977) abarcaba los yacimien- 
tos maastrichtienses situados entre los ríos Noguera Pallaresa y Noguera-Ribargoçana y contenía la descripción de 27 especies de equínidos y la identificación de la fauna acompañante, presentando su distribución en 21 series estratigráficas; Gallemí (1979), resumió y discutió la distribución, conservación y abudancia de esta fauna. Gallemí (1982), presentó y comentó la distribución de los equínidos del Cretácico superior surpirenaico.

En su tesis doctoral, Gallemí (1993), describió 60 especies de equínidos del Cretácico superior surpirenaico (23 citadas por primera vez en la zona), incluyendo dibujos a cámara clara de perfiles y sistemas apicales y ambulacrales, así como gráficas de medidas y discutiendo las citas anteriores de especies no contenidas en su estudio. Como conclusión taxonómica hay que destacar que pudo determinarse la evolución de las especies del género Micraster para el intervalo Coniaciense-Campaniense. La distribución de los equínidos le permitió establecer 4 cenozonas desde el Cenomaniense al Maastrichtiense inferior. Para el intervalo de cada cenozona, comparó la fauna pirenaica con la de otras regiones constatando que la más amplia distribución paleobiogeográfica corresponde a las especies del Cenomaniense inferior y que existe una notable coincidencia para las del Campaniense y Maastrichtiense no sólo con áreas del Reino Templado Septentrional sino también con zonas del Tethys oriental.

\section{ESTUDIOS INTEGRADOS}

En varias ocasiones se ha intentado integrar la distribución de distintos grupos fósiles dentro del mismo contexto geológico. Así, Caus y Gómez-Garrido (1989a y 1989b) y Caus et al. (1992, 1993 y 1994) correlacionaron los foraminíferos bentónicos y planctónicos; Gallemí, Martínez y Pons (1983) y Pascual (1987) consideraron la distribución conjunta de diversos grupos de macrofauna y en Caus et al. (1981) se estableció la relación entre microfauna y macrofauna. En la figura 2 se presenta un cuadro resumen comparativo de las biozonas y/o asociaciones de los diversos grupos faunísticos reconocidas en algunos de los trabajos citados.

La necesidad de contar con una base cartográfica y un marco litoestratigráfico adecuado donde situar la distribución de los fósiles hizo que se elaborara (Gallemí, Martínez y Pons 1982) una subdivisión litoestratigráfica a nivel de formaciones y miembros aplicable a los materiales del Cretácico superior de los alrededores de Sant Corneli. Bastantes de estas unidades se incorporaron como término habitual a la literatura geológica pirenaica en trabajos de síntesis (p. ej. Souquet, 1984 y 1986) y dado que los límites entre algunas de ellas correspondían a discontinuidades sedimentarias, cuando se empezó a aplicar la estratigrafía secuencial al estudio del Cretácico superior pirenaico, algunas de las unidades litoestratigráficas mayores pasaron a representar

\begin{tabular}{|c|c|c|c|c|c|c|c|c|c|c|}
\hline \multirow{2}{*}{\multicolumn{2}{|c|}{ Pisos }} & Ammonites & $\begin{array}{l}\text { Foraminíferos } \\
\text { planctónicos }\end{array}$ & Inocerámidos & \multicolumn{4}{|c|}{ Rudistas } & Equínidos & $\begin{array}{l}\text { F. bentónicos } \\
\text { gen. Orbitoides }\end{array}$ \\
\hline & & 1 & 2 & 3 & 4 & 5 & 6 & 7 & 8 & 9 \\
\hline \multirow[t]{3}{*}{ MAASTRICHT. } & sup. & & gansseri & & & & & & & \\
\hline & inf & nouboraich & & \multirow{2}{*}{$8^{a}$} & A & \multirow[b]{2}{*}{ Maas. } & $\mathrm{Ma2}$ & & \multirow{2}{*}{ Maas. inf. } & \multirow{2}{*}{ gruembachensis } \\
\hline & Int. & neubergicus & falsostuarti & & & & Mal & $\mathrm{Mal}$ & & \\
\hline \multirow{3}{*}{ CAMPANIENSE } & sup. & polyplocum & calcarata & $7^{a}$ & B & \multirow{3}{*}{ Camp. } & & Ca-Ma & \multirow{3}{*}{ Camp. } & megaloformis \\
\hline & med. & & ventricosa & $6^{a}$ & & & $\mathrm{Ca} 2$ & $\mathrm{Ca} 2$ & & media \\
\hline & $\inf$ & & elevata & $5^{a}$ & c & & Cal & Cal & & douvillei \\
\hline \multirow{3}{*}{ SANTONIENSE } & sup. & depressum & \multirow[b]{2}{*}{ asymetrica } & $4^{a}$ & & \multirow{3}{*}{ Sant. } & & & \multirow{4}{*}{$\begin{array}{c}\text { Sant- } \\
\text { Con. sup. }\end{array}$} & \\
\hline & med. & lapparenti & & $3^{a}$ & $\mathrm{D}$ & & Sa2 & $\mathrm{Sa} 2$ & & hottingeri \\
\hline & inf. & texanus & \multirow[b]{2}{*}{ concavata } & $2^{a}$ & \multirow[b]{2}{*}{ E } & & Sal & & & \\
\hline \multirow{2}{*}{ CONIACIENSE } & sup. & emscheris & & $1^{a}$ & & \multirow{2}{*}{ Con. } & \multirow{2}{*}{ Co } & & & \\
\hline & inf. & petrocoriensis & schneegansi & & & & & & & \\
\hline \multirow{3}{*}{ TURONIENSE } & sup. & & sigali & & & \multirow{3}{*}{ Tur. } & $\mathrm{Tu}$ & & & \\
\hline & med. & woollgari & helvetica & & & & & & & \\
\hline & inf. & & cretacea & & & & & & & \\
\hline \multirow{3}{*}{ CENOMANIENSE } & sup. & & & & & \multirow{3}{*}{ Cen. } & & & & \\
\hline & med. & rhotomagense & cushmani & & & & & & & \\
\hline & inf. & mantelli & brotzeni & & & & & & Cen. inf. & \\
\hline
\end{tabular}

Figura 2. Cuadro resumen comparativo de las biozonas y/o asociaciones reconocidas con los diversos grupos faunísticos en algunos de los trabajos comentados en el texto 1, Martínez (1982a y 1982b). 2, Gómez-Garrido (1989) y Caus et al. (1993). 3, López (1986a y 1986b). 4, Pons (1977). 5, Pons (1982). 6, Pascual, Pons y Vicens (1989). 7, Vicens (1992). 8, Gallemí (1993). 9, Caus, Bernaus y Gómez-Garrido (1994). 
secuencias deposicionales (ver Simó 1986; Puigdefábregas y Souquet 1986).

Los datos bioestratigráficos y paleoecológicos obtenidos en los trabajos paleontológicos comentados han sido fundamentales en el momento de establecer la edad de las secuencias deposicionales del Cretácico superior del Pirineo.

Por último, algunos de los fósiles del Cretácico pirenaico estudiados en estos trabajos han sido utilizados para ilustrar trabajos de divulgación científica (ver Gómez-Alba, 1988 y Gallemí, 1988).

\section{AGRADECIMIENTOS}

Naturalmente, esta revisión no hubiera sido posible sin el trabajo previo de todos los autores citados. El Servei Geològic de Catalunya contribuyó en diversos aspectos a la realización de algunos de los trabajos comentados, así como otros se beneficiaron de la financiación de los proyectos de la DGICYT: PB-85- 0082, PB-85-0156 y PB-90-0716.

\section{BIBLIOGRAFÍA}

Caus, E. 1988. Upper Cretaceous Larger Foraminifera: Paleoecological Distribution. Revue de Paléobiologie. Volume Spécial, 2 (Benthos '86), 417-419.

Caus, E., Bernaus, J. M. y Gómez-Garrido, A. 1994. Reevaluación de las especies del género Orbitoides y su uso en bioestratigrafía. In: Comunicaciones de las X Jornadas de Paleontología (Coord. S. Fernández López), Depto. de Paleontología (UCM), UEI de Paleontología (CSIC-UCM) y Sociedad Española de Paleontología, Madrid, 43-45.

Caus, E. y Cornella, A. 1981a. Estructura de Montsechiana montsechiensis n. sp., foraminífero del Cretácico superior sudpirenaico. Revista Española de Micropaleontología, 13 (2), 201-212.

Caus, E. et Cornella, A. 1981b. Calveziconus lecalvezae n. gen. n. sp., orbitolinidé campanien de la bordure méridionale des Pyrénées. Cahiers de Micropaléontologie, 4, 27-34.

Caus, E. et Cornella, A. 1983. Macroforaminiféres du Crétacé supérieur du bassin sud-pyrénéen. Géologie Méditerranéenne, 10 (3-4), 137-142.

Caus, E., Cornella, A., Gallemí, J., Gili, E., Martínez, R. and Pons, J. M. 1981. Field Guide: Excursions to Coniacian-Maastrichtian of South Central Pyrenees. Publicaciones de Geología, Universidad Autónoma de Barcelona, 13, 1-70.

Caus, E., Cornella, A. y Gómez-Garrido, A. 1982. Evolución de la cuenca sedimentaria del Cretácico superior surpirenaico entre los ríos Segre y Noguera Ribagorzana (NE España). Cuadernos de Geología Ibérica, 8, 965-977.

Caus, E., Cornella, A. y Pons, J. M. 1978. Foraminíferos bentónicos del Santoniense sudpirenaico (Montsec de Rubies, Prov. de Lérida, España). Nueva adscripción genérica de Dictyopsella chalmasi Schlumberger. Revista española de Micropaleontología, 10 (3), 453-460.

Caus, E. and Gómez-Garrido, A. 1989a. Correlation of Larger Benthic and Planktonic Foraminifera of the Late Cretaceous in the South-Central Pyrenees. In: Cretaceous of the Western Tethys. Proceedings 3rd International Cretaceous Symposium, Tübingen (Ed. J. Wiedmann). E. Schweizerbart'sche Verlagsbuchhandlung, Stuttgart, 231-238.
Caus, E. and Gómez-Garrido, A. 1989b. Upper cretaceous biostratigraphy of the south-central Pyrenees (Lleida, Spain). Geodinamica Acta, 3 (3), 221-228.

Caus, E., Gómez-Garrido, A. and Rodés, D. 1988. Reevaluation of Lepidorbitoides evolution in function of the age relation between species as established with Nannoplankton biostratigraphy. Revue de Paléobiologie. Volume Spécial 2 (Benthos '86), 421-428.

Caus, E., Gómez-Garrido, A., Simó, A. y Soriano, K. 1992. Los sedimentos del Cenomaniense-Turoniense en el Surpirineo Central (Lleida, Huesca). III Congreso Geológico de España. Simposios, 2, 68-76.

Caus, E., Gómez-Garrido, A., Simó, A. and Soriano, K. 1993. Cenomanian-Turonian platform to basin integrated stratigraphy in the South Pyrenees (Spain). Cretaceous Research, 14, 531-551.

Caus, E. y Hottinger, L. 1986. Particularidades de la fauna (macroforaminíferos) del Cretácico superior pirenaico. Paleontologia i Evolució, 20, 115-123.

Caus, E., Rodés, D. y Solé-Sugrañes, L. 1988. Bioestratigrafía y estructura del Cretácico superior de la Vall d'Alinyà (Pirineo oriental, prov. de Lleida). Acta Geológica Hispánica, 23 (2), 107-118.

Caus, E. y Serra-Kiel, J. 1984. Distribución ambiental de los macroforaminíferos: Cretácico superior y Eoceno. I Congreso Español de Geología, 1, 399-406.

Caus, E. y Vicens, E. 1985. La fauna cretácica del Castell de Bac Grillera (Pirineos Catalanes). Acta Geologica Hispanica, 19 (4) (1984), 267-276.

Conesa, J. A. 1979. Foraminíferos del Cretácico superior del sinclinal de Carreu (prov. de Lérida). Publicaciones de Geología. Universidad Autónoma de Barcelona, 10, 1-64, 6 láms.

Cornella, A. 1977. Foraminíferos bentónicos del Santoniense del bco. de la Font de la Plata. Montsec de Rubies (prov. de Lérida). Publicaciones de Geología. Universidad Autónoma de Barcelona, 8, 1-45, 3 láms.

Gallemí, J. 1977. Los yacimientos con equínidos del nivel "Homes Morts" entre los ríos N. Pallaresa y N. Ribagorzana (Cret. sup. Prepirineo de Lérida). Publicaciones de Geología, Universidad Autónoma de Barcelona, 6, 1-92, 12 láms.

Gallemí, J. 1979. Equínidos cretácicos del nivel "Homes Morts" entre los ríos N. Pallaresa y N. Ribagorzana (Provincia de Lérida). Cuadernos de Geología Ibérica, 5, 353-363.

Gallemí, J. 1982. Distribución de los equínidos del Cretácico superior sudpirenaico. Cuadernos de Geología Ibérica, 8, 1049-1056.

Gallemí, J. (coord.) 1988. Paleontologia. In: Història Natural dels Països Catalans, 15 Registre fòssil. Enciclopèdia Catalana, Barcelona, 74-429.

Gallemí, J. 1993. Los yacimientos con equínidos del Cretácico superior del Prepirineo de la provincia de Lleida. Tesis de Doctorado, Facultad de Ciencias, Universidad Autónoma de Barcelona.

Gallemí, J. y Calzada, S. 1979. "Rhynchonella" coquandi n. sp. (Maastrichtiense) y su posición estratigráfica. Acta Geologica Hispanica, 13 (1) (1978), 5-9.

Gallemí, J., Martínez, R. y Pons, J. M. 1982. Unidades del Cretácico superior en los alrededores de Sant Corneli (Provincia de Lleida). Cuadernos de Geología Ibérica, 8, 935-948.

Gallemí, J. Martínez, R. and Pons, J. M. 1983. Coniacian - Maastrichtian of the Tremp Area (South Central Pyrenees). Newsletters on Stratigraphy, 12 (1), 1-17. 
Gili, E. 1982. Análisis sedimentológico e interpretación ambiental de una formación de rudistas (Santoniense, zona surpirenaica). Cuadernos de Geología Ibérica, 8, 979-986.

Gili, E. 1983. Interaccions sedimentològiques i biològiques a les formacions calcàries de rudistes (Bivalvia) de les Collades de Basturs. Tesis de doctorado, Facultad de Ciencias, Universidad Autónoma de Barcelona. [Resumen publicado en 1984. Publicacions de la Universitat Autónoma de Barcelona, Bellaterra, 42 pp.]

Gili, E. 1985. Les Collades de Basturs. In: Transition from shelf to basin on an active slope, Upper Cretaceous, Tremp area, southern Pyrenees (A. Simó and C. Puigdefabregas). In: 6th European Regional Meeting, Excursion Guidebook (Eds. M. D. Milá and J. Rosell). Institut d'Estudis Ilerdencs, Lleida, 78-81.

Gili, E. 1992a. Palaeoecological significance of rudist constructions: a case study from les Collades de Basturs (Upper Cretaceous, south-central Pyrenees). Geologica Romana, 28, 319-325.

Gili, E. 1992b. Day 2, stop 3. Locality: les Collades de Basturs. In: Field guide. Upper Cretaceous carbonate platforms, southcentral Pyrenees (E. Gili, D. Rodrigues-de-Miranda and J. A. Simó). Workshop of CRER Working Group 4. GSGP, 23-30.

Gili, E. 1993. Facies and geometry of les Collades de Basturs carbonate platform, Upper Cretaceous, south-central Pyrenees. In: Cretaceous carbonate platforms (Eds. J. A. Simó, R. W. Scott \& J. P. Masse). American Association of Petroleum Geologists, Memoir 56, 343-352.

Gili, E. and Pons, J. M. 1983. Collades de Basturs: a rudist platform model (Upper Cretaceous, South Central Pyrenees). First International Congress on Paleoecology. Abstracts, 73.

Gili, E. y Pons, J. M. 1988. Distribución ecológica de los Rudistas (Bivalvia) en les Collades de Basturs. Congreso Geológico de España 88, Comunicaciones, 1, 285-288.

Gili, E., Pons, J. M. y Vicens, E. 1986. Problemática del uso de los Rudistas (Bivalvia) en Bioestratigrafía. In: Memorias de las I Jornadas de Paleontología (Coord. E. Villas), Diputación General de Aragón, Zaragoza, 121-130.

Gili, E., Pons, J. M. and Vicens, E. 1987. Rudist distribution and provinciality in the Mediterranean Tethys: a critique of the data base. Terra Cognita, 7, 104.

Gómez-Alba, J. 1988. Guía de Campo de los fósiles de España y de Europa. Ediciones Omega, S.A., Barcelona, 925 pp.

Gómez-Garrido, A. 1981. Foraminíferos planctónicos de la Formación Reguard (Turoniense) en el valle del Flamicell (Prepirineo de Lleida). Publicaciones de Geología. Universidad Autónoma de Barcelona, 16, 1-48, 3 láms.

Gómez-Garrido, A. 1988. Foraminíferos planctónicos del Cretácico superior del Surpirineo central. Tesis de Doctorado, Facultad de Ciencias, Universidad Autónoma de Barcelona.

Gómez-Garrido, A. 1989. Bioestratigrafía (Foraminíferos planctónicos) del Cretácico superior del Surpirineo central (España). Revista Española de Micropaleontología, 21 (1), 145- 171.

Hottinger, L. and Caus, E. 1982. Marginoporiform structure in Ilerdorbis decussatus n. gen. n. sp., a Senonian, agglutinated, discoidal foraminifer. Eclogae geologicae Helveticae, 75 (3), 807-819.

Hottinger, L. and Caus, E. 1993. Praestorrsella roestae (Visser), a foraminiferal index fossil for Late Cretaceous deeper neritic deposits. Zitteliana, 20, 213-221.
Hottinger, L., Drobne, K. and Caus, E. 1989. Late Cretaceous, larger, complex miliolids (Foraminifera) endemic in the Pyrenean faunal Province. Facies, 21, 99-134.

López, G. 1986a. Inocerámidos del Cretácico superior de los alrededores de Sant Corneli (Prov. Lleida). Publicaciones de Geología, Universitat Autónoma de Barcelona, 22, 1-123.

López, G. 1986b. Distribución de Inocerámidos (Bivalvia) en la zona sudpirenaica central. Paleontologia i Evolució, 20, 235239, 1 lám.

López, G. En prensa. Els inoceràmids (Bivalvia) del Cretaci superior del Montsec d'Ares (Lleida). Métode d'estudi i descripció de les espècies. Ilerdia, Ciència.

López, G., Martínez, R. \& Lamolda, M. A. 1992. Biogeographic relationships of the Coniacian and Santonian inoceramids bivalves of northern Spain. Palaeogeography, Palaeoclimatology, Palaeoecology, 92, 249-261.

Mañas, D. 1977. Microfauna de la Fm. Anseroles (Senoniense) en los alrededores de St. Corneli (prov. de Lérida). Publicaciones de Geología. Universidad Autónoma de Barcelona, 4, 1-58, 1 lám.

Mañas, D., Caus, E. i Pons, J. M. 1979. Foraminífers de les "Margues amb Micraster" (Cretacic superior) de les rodalies de l'anticlinal de Sant Corneli (Lleida). Butlletí de la Instituciò Catalana d'Història Natural, 43, 101-110.

Martínez, R. 1977. Macrofauna de la Fm Margas de Llucá (Apt.Alb.) entre los ríos Flamicell y Noguera Pallaresa (Prov. de Lérida). Publicaciones de Geología, Universidad Autónoma de Barcelona, 5, 1-82, 10 láms.

Martínez, R. 1979. Cefalópodos de la formación "Margas de Llucá" (Apt.-Alb.) al norte de Pobla de Segur (prov. de Lérida). Cuadernos de Geología Ibérica, 5, 339-351.

Martínez, R. 1982a. Ammonoideos cretácicos del Prepirineo de la provincia de Lérida. Publicaciones de Geología, Universidad Autónoma de Barcelona, 17, 1-197, 30 láms.

Martínez, R. 1982b. Distribución de los ammonites del Cretácico sudpirenaico. Cuadernos de Geología Ibérica, 8, 1035-1047.

Martínez, R. y Vicens, E. 1987-88. Ammonites del Campaniense y del Maastrichtiense del Bergadà (Prepirineo Catalán). Revista d'Investigacions Geològiques, 44/45, 415-429.

Muñoz, J. 1985. Braquiópodos del Cretácico superior de los alrededores de Sant Corneli (Prov. Lleida). Publicaciones de Geología, Universitat Autònoma de Barcelona, 21, 1-125.

Muñoz, J. 1990. Microbraquiópodos del Campaniense-Maastrichtiense de los alrededores de Berga (Prepirineo, provincia de Barcelona). Boletín de la Real Sociedad Española de Historia Natural (Geología), 85, 55-81.

Muñoz, J. 1993. Estudio paleontológico y bioestratigráfico de los braquiópodos del Cretácico superior del Sudpirineo Catalán. Tesis de Doctorado, Facultad de Ciencias, Universidad Autónoma de Barcelona.

Muñoz, J. 1994. Un nuevo Thecidellininae (Brachiopoda) monoseptal del Santoniense de la Serra del Montsec (Surpirineo central, NE España). Revista Española de Paleontología, 9 (2), 165-175.

Pascual, O. 1987. El Cretácico superior entre Abella y Boixols (prov. Lleida). Publicaciones de Geología, Universitat Autònoma de Barcelona, 23, 1-91.

Pascual, O., Pons, J. M. and Vicens, E. 1989. Rudist horizons in the Montsec (South Central Pyrenees). In: Cretaceous of the Western Tethys. Proceedings 3rd International Cretaceous 
Symposium, Tübingen, 1987 (Ed. J. Wiedmann). E. Schweizerbart'sche Verlagsbuchhandlung, Stuttgart, 215-230.

Philip, J., Bilotte, M. et Pons, J. M. 1983. Rudistes. In: Conclusions au Colloque sur les étages Coniacien a Maastrichtien: Echelles biostratigraphiques. Géologie Méditerranéenne, 10 (3-4), 424-426.

Pons, J. M. 1977. Estudio estratigráfico y paleontológico de los yacimientos con rudístidos del Cretácico superior del Prepirineo de la Provincia de Lérida. Publicaciones de Geología, Universidad Autónoma de Barcelona, 3, 1-103, 87 láms.

Pons, J. M. 1982. Distribución de los Rudistas (Bivalvia) del Cretácico superior sudpirenaico. Cuadernos de Geología Ibérica, $\mathbf{8}$, 1027-1033.

Pons, J. M. and Sirna, G. 1992. Upper Cretaceous Rudists distribution in the Mediterranean Tethys: Comparison between platforms from Spain and South Central Italy. Geologica Romana, 28, 341-349.

Pons, J. M. y Vicens, E. 1986. Nuevos datos sobre Biradiolites chaperi (Bivalvia: Radiolitidae) del Maastrichtiense. Revista d'Investigacions Geològiques, 42/43, 67-75.

Pons, J. M. and Vicens, E. 1991 en prensa. Campanian and Maastrichtian Rudists from southern Valencia province, South East Spain. In: Proceedings First International Conference on Rudists (Beograd, 1988) (Ed. M. Sladic). Serbian Geological Society, Special Publication 2, Beograd, 1-31.

Puigdefábregas, C. and Souquet, P. 1986. Tecto-sedimentary cycles and depositional sequences of the Mesozoic and Tertiary from the Pyrenees. Tectonophysics, 129, 173-203.

Rodés, D. 1983. Bioestratigrafia del Cretaci superior de la vall del riu Perles (Lleida). Tesis de Licenciatura, Facultad de Ciencias, Universidad Autónoma de Barcelona.

Sánchez, M. V. 1981. Hippuritidae y Radiolitidae (Bivalvia). Catálogo de Especies. Publicaciones de Geología, Universidad Autónoma de Barcelona, 15, 1-228.
Scott, R. W., Fernández-Mendiola, P. A., Gili, E. and Simó, A. 1990. Persistence of Coral-Rudist Reefs into the Late Cretaceous. Palaios, 5, 98-110.

Simó, A. 1986. Carbonate platform depositional sequences. Upper Cretaceous, south-central Pyrenees (Spain). Tectonophysics, 129, 205-231.

Souquet, P. 1984. Les cycles majeurs du Crétacé de la paléomarge ibérique dans les Pyrénées. Strata, 1, 47-70.

Souquet, P. 1986. El cicle cretaci superior als Pirineus. In: Història Natural dels Països Catalans, 1 Geologia (1). Enciclopèdia Catalana, Barcelona, 375-396.

Teixell, A. 1992. Estructura alpina en la transversal de la terminación occidental de la zona axial pirenaica. Tesis de Doctorado, Facultad de Geología, Universidad de Barcelona.

Vicens, E. 1984. Los yacimientos con rudistas del Cretácico superior de l'Alt Empordà. Publicaciones de Geología, Universitat Autònoma de Barcelona, 19, 1-85, 14 láms.

Vicens, E. 1992a. Estudio de la fauna de rudistas (Hippuritidae y Radiolitidae) de los materiales cretácicos del Pirineo oriental: Implicaciones bioestratigráficas. Tesis de Doctorado, Facultad de Ciencias, Universidad Autónoma de Barcelona.

Vicens, E. 1992b. Intraspecific variability in Hippuritidae in the southern Pyrenees, Spain: Taxonomic implications. Geologica Romana, 28, 119-161.

Vidal, A. 1980. Los Scleractinia de Collades de Basturs (Con.- Sant. Prepirineo de la Provincia de Lérida). Publicaciones de Geología, Universidad Autónoma de Barcelona, 11, 1-94, 12 láms.
Manuscrito recibido: 26 de enero, 1995 Manuscrito aceptado: 20 de septiembre, 1995 